\title{
Multiple solutions to Neumann problems with indefinite weight and bounded nonlinearities
}

\author{
Alberto Boscaggin and Maurizio Garrione
}

\begin{abstract}
We study the Neumann boundary value problem for the second order ODE

$$
u^{\prime \prime}+\left(a^{+}(t)-\mu a^{-}(t)\right) g(u)=0, \quad t \in[0, T],
$$

where $g \in \mathcal{C}^{1}(\mathbb{R})$ is a bounded function of constant sign, $a^{+}, a^{-}:[0, T] \rightarrow$ $\mathbb{R}^{+}$are the positive/negative part of a sign-changing weight $a(t)$ and $\mu>$ 0 is a real parameter. Depending on the sign of $g^{\prime}(u)$ at infinity, we find existence/multiplicity of solutions for $\mu$ in a "small" interval near the value

$$
\mu_{c}=\frac{\int_{0}^{T} a^{+}(t) d t}{\int_{0}^{T} a^{-}(t) d t} .
$$

The proof exploits a change of variables, transforming the sign-indefinite equation (1) into a forced perturbation of an autonomous planar system, and a shooting argument. Nonexistence results for $\mu \rightarrow 0^{+}$and $\mu \rightarrow+\infty$ are given,
\end{abstract} as well.

AMS-Subject Classification. 34B15; 34B08.

Keywords. Indefinite weight; Bounded nonlinearities; Neumann problem; Shooting method.

\section{Introduction and statement of the main results}

In this paper, we investigate the Neumann problem for the second order ordinary differential equation

$$
u^{\prime \prime}+q(t) g(u)=0, \quad t \in[0, T],
$$

where $g \in \mathcal{C}^{1}(\mathbb{R})$ is a bounded function of constant sign. By integrating the equation on $[0, T]$, a necessary solvability condition is thus immediately found: the weight function $q(t)$ has to change its sign in $[0, T]$, that is, $q(t)$ is an indefinite weight. This terminology goes back at least to [2], where it was introduced dealing with a linear eigenvalue problem, and it has become very popular starting with the paper [16] by Hess and Kato. 
In spite of the wide bibliography dealing with nonlinear boundary value problems associated with the scalar equation

$$
u^{\prime \prime}+f(t, u)=0
$$

with $f:[0, T] \times \mathbb{R} \rightarrow \mathbb{R}$ a bounded function, the case which we are taking into account (namely, $f(t, u)=q(t) g(u)$ with the above assumptions on the sign of $q(t)$ and $g(u))$ seems to be quite new. It is worth noticing that classical nonresonance assumptions (recall that the boundedness of $f(t, u)$ implies that the Neumann problem for equation (3) is resonant with respect to the principal eigenvalue $\lambda_{0}=0$ ) do not apply in this setting. For instance, the well-known Ahmad-Lazer-Paul conditions $^{1}$ (see $[13,18]$ )

$$
\lim _{|u| \rightarrow+\infty} \int_{0}^{T} F(t, u) d t=-\infty \quad \text { or } \quad \lim _{|u| \rightarrow+\infty} \int_{0}^{T} F(t, u) d t=+\infty
$$

fail for equation (2) (here $F(t, u)=\int_{0}^{u} f(t, s) d s$ ), since in this case

$$
\int_{0}^{T} F(t, u)=\left(\int_{0}^{T} q(t) d t\right) G(u), \quad \text { with } \quad G(u)=\int_{0}^{u} g(s) d s,
$$

and the fact that $g(u)$ never vanishes implies that either $\operatorname{sgn}(u) G(u)>0$ for every $u \neq 0$ or $\operatorname{sgn}(u) G(u)<0$ for every $u \neq 0$.

It is the aim of the present paper to show that the existence of Neumann solutions to (2) may actually depend in a quite subtle way on the interplay between the weight function $q(t)$ and the nonlinearity $g(u)$ at infinity. To achieve this goal, we impose some specific conditions both on the nonlinear term and on the weight function, but, on the other hand, we manage to obtain quite accurate conclusions.

The basic assumption which is required henceforth for $g(u)$ is that, for a suitable $d>0$,

$$
g^{\prime}(u) \neq 0 \text { for }|u|>d, \quad \text { and } \quad \lim _{|u| \rightarrow+\infty} g^{\prime}(u)=0
$$

Hence, various cases can be taken into account according to the values of

$$
\operatorname{sgn}(g(u)), \quad \operatorname{sgn}\left(\left.g^{\prime}(u)\right|_{\{u<-d\}}\right), \quad \operatorname{sgn}\left(\left.g^{\prime}(u)\right|_{\{u>d\}}\right) .
$$

However, as it will be clear from the proofs, it is not restrictive to assume that the first two terms in the line above are positive, thus focusing on the sign of $g^{\prime}(u)$ for

\footnotetext{
${ }^{1}$ These assumptions are related to the variational approach to (3) and imply, respectively, the coercivity or a saddle geometry for the associated Lagrange functional (other conditions coming from topological degree theory, like the Landesman-Lazer ones, could be given, as well, being however less general [13]). Notice that, usually, nonresonance conditions are stated for the $T$ periodic problem, but the same results hold when one considers Neumann solutions. Our choice of studying the Neumann BVP is due to the (shooting) method used in our proofs.
} 
$u>d$ (indeed, the other combinations can be treated with similar arguments). As two possible models, we can take for instance

$$
g(u)=\arctan (u)+\frac{\pi}{2},
$$

for the case $g^{\prime}(u)>0$ for $u \gg 0$, and

$$
g(u)=\frac{1}{1+u^{2}},
$$

for the case $g^{\prime}(u)<0$ for $u \gg 0$. Notice that, in both the above examples, the limits $g( \pm \infty)$ exist (and $g(-\infty)=g(+\infty)$ in the second one); however, this is not essential (see also the first remark after the statements of Theorems 1.1 and 1.2).

As for the weight function, the essential point is to introduce and exploit the following dependence on a real parameter: we take

$$
q(t)=q_{\mu}(t)=a^{+}(t)-\mu a^{-}(t),
$$

where $\mu>0$ is the parameter and $a^{+}(t), a^{-}(t)$ are the positive and the negative part of a sign-changing function $a \in L^{1}(0, T)$ (accordingly, the fact that $a(t)$ is signchanging is understood up to zero measure sets, i.e., $\int_{0}^{T} a^{+}(t) d t, \int_{0}^{T} a^{-}(t) d t>0$, and solutions to the differential equations are meant in the Carathéodory sense). We also make the further assumption (suggested by the method used in the proof, compare with [8]) that $a(t)$ changes sign just once on $[0, T]$, that is (up to substituting $T-t$ for $t$ ) there exists $\tau \in[0, T]$ such that

$$
a(t) \geq 0 \text { for a.e. } t \in[0, \tau], \quad a(t) \leq 0 \text { for a.e. } t \in[\tau, T] .
$$

It is worth noticing that indefinite weights of the type (6) were already considered in $[6,12,14,15]$, in connection with the existence of positive solutions to the Dirichlet problem for the equation $u^{\prime \prime}+q_{\mu}(t) u^{p}=0$ (and its PDE counterpart), in the superlinear case $p>1$. However, while in those papers solutions are found for $\mu \gg 0$, here existence and multiplicity can be guaranteed when the parameter is "near" (the precise picture depending on the sign of $g^{\prime}(u)$ at $+\infty$ )

$$
\mu_{c}=\frac{\int_{0}^{T} a^{+}(t) d t}{\int_{0}^{T} a^{-}(t) d t},
$$

this being the value of $\mu$ such that $q_{\mu}(t)$ has zero average. Of course, this restriction requires some comments and motivations; we will discuss in detail this point later (see the second remark after the statements of Theorems 1.1 and 1.2, and Section 4).

Let us now come to the precise statements. Consider the problem

$$
\left\{\begin{array}{l}
u^{\prime \prime}+\left(a^{+}(t)-\mu a^{-}(t)\right) g(u)=0 \\
u^{\prime}(0)=u^{\prime}(T)=0
\end{array}\right.
$$

namely, the differential equation (2), with $q(t)$ as in (6), together with Neumann boundary conditions on $[0, T]$. We first take into account the case when the nonlinear term is strictly increasing for $u$ positive and large, like (4). 
Theorem 1.1. Assume that $g \in \mathcal{C}^{1}(\mathbb{R})$ is a positive function such that, for a suitable $d>0$,

$$
g^{\prime}(u)>0 \quad \text { for }|u|>d,
$$

and

$$
\lim _{|u| \rightarrow+\infty} g^{\prime}(u)=0 .
$$

Moreover, suppose that $a \in L^{1}(0, T)$ satisfies (7). Then, there exists $\mu^{*}>\mu_{c}$ such that, for every $\mu \in] \mu_{c}, \mu^{*}[$, problem (9) has at least two solutions.

We now give the statement for nonlinearities strictly decreasing for $u$ positive and large, like (5).

Theorem 1.2. Assume that $g \in \mathcal{C}^{1}(\mathbb{R})$ is a positive function such that, for $a$ suitable $d>0$,

$$
\operatorname{sgn}(u) g^{\prime}(u)<0 \quad \text { for }|u|>d
$$

and

$$
\lim _{|u| \rightarrow+\infty} g^{\prime}(u)=0 .
$$

Moreover, suppose that $a \in L^{1}(0, T)$ satisfies (7). Then, there exist $\mu_{*}, \mu^{*}>0$ with $\mu_{*}<\mu_{c}<\mu^{*}$ such that problem (9) has at least one solution for $\mu=\mu_{c}$ and two solutions for $\mu \in] \mu_{*}, \mu^{*}\left[\backslash\left\{\mu_{c}\right\}\right.$.

We mention that our original motivation for the study of nonlinearities like $g(u)=1 /\left(1+u^{2}\right)$ were the papers $[4,11,22]$, dealing with the solvability of the (resonant) $T$-periodic problem associated with $u^{\prime \prime}+g(u)=s+p(t)$, with $p(t)$ a $T$ periodic function and $s$ a positive parameter. In those works, an Ambrosetti-Prodi type result is proved, according to the classical scheme zero/one/two solutions for $s$ belonging to different ranges of values. Theorem 1.2 shows that, for the indefinite problem (9), a somewhat similar picture is obtained. Also, our results suggest some analogies with the ones recently obtained in [7] for the existence/multiplicity of positive solutions to super-sublinear problems, possibly deserving future attention.

A few remarks are now in order.

(i) The boundedness of $g(u)$ (which is assumed throughout the Introduction for the sake of simplicity) actually is not necessary. However, in view of $(\diamond)$, it turns out that $g(u)$ is sublinear at infinity, i.e., $g(u) / u \rightarrow 0$ for $|u| \rightarrow+\infty$.

(ii) The condition $\mu>\mu_{c}$ (i.e., $\int_{0}^{T}\left(a^{+}(t)-\mu a^{-}(t)\right) d t<0$ ) in Theorem 1.1 cannot be removed, in general. Indeed, it is necessary for the solvability whenever $g^{\prime}(u)>0$ for any $u \in \mathbb{R}$ (see Remark 3.2). Notice that this restriction does not appear in Theorem 1.2 since the sign condition $\operatorname{sgn}(u) g^{\prime}(u)<0$ for $|u|$ large automatically rules out strictly increasing nonlinearities. On the other hand, nonexistence results can often be proved for $\mu \rightarrow+\infty$ in Theorem 1.1 and both for $\mu \rightarrow 0^{+}$and $\mu \rightarrow+\infty$ in Theorem 1.2, showing that, also from this point of view, the above statements cannot be improved. We refer to Section 4 for further details. 
(iii) As it will be clear from the proofs, in place of (6) one could consider weight functions of the form $q_{\lambda}(t)=\lambda a^{+}(t)-a^{-}(t)$, so as to prove corresponding results for $\lambda$ near the value $\lambda_{c}=\mu_{c}^{-1}$.

(iv) In a standard way, Theorems 1.1 and 1.2 can be used to construct $T$-periodic solutions to (2) (with $q(t)$ as in (6)) when the weight function $a(t)$ is $T$ periodic, satisfies $a(\sigma+t)=a(\sigma-t)$ for some $\sigma \in[0, T[$ and almost every $t \in \mathbb{R}$ and, for a suitable $\left.\tau^{\prime} \in\right] 0, T / 2[$,

$$
a(t) \geq 0 \text { for a.e. } t \in\left[\sigma, \sigma+\tau^{\prime}\right], \quad a(t) \leq 0 \text { for a.e. } t \in\left[\sigma+\tau^{\prime}, \sigma+T / 2\right],
$$

(notice that this is always the case if the weight function $a(t)$ is piecewise constant). Indeed, one first solves the Neumann BVP on $[\sigma, \sigma+T / 2]$ and then finds a $T$-periodic solution extending by symmetry with respect to $\sigma$ and by $T$-periodicity (cf. [8, Corollary 4]). Also, it is possible to use our results to construct radial solutions to the PDE counterpart of (9) on an annulus, when the weight function is radial.

The proofs of Theorems 1.1 and 1.2 rely on an elementary shooting argument. First, we use the change of variables introduced in [8] to transform (2) into a first order planar system of the type

$$
\left\{\begin{array}{l}
x^{\prime}=y \\
y^{\prime}=h(x) y^{2}+q(t)
\end{array}\right.
$$

converting the original Neumann problem into the search for solutions to (10) satisfying $y(0)=y(T)=0$. Such solutions are in a one-to-one correspondence with the intersections between the two planar curves obtained by shooting the $x$-axis forward (from 0 to $\tau$ ) and backward (from $T$ to $\tau$ ) in time (this indeed motivates the choice of a two-step function $a(t)$ as in (7); we do not exclude, in principle, that more general configurations could be considered at the expense of longer computations). Thus, taking into account that $q(t)=a^{+}(t)-\mu a^{-}(t)$, the crucial point is to carefully understand how the mutual position of such curves changes in dependence on the parameter, so as to identify the ranges of $\mu$ for which they actually intersect.

The reason why we study (10) instead of (2) in the standard phase-plane lies in the fact that the dynamics in the latter case appears much more difficult to decode (see the numerical simulations in Section 4); thus, the possibility of changing the original sign-indefinite equation (2) into a forced perturbation of an autonomous planar system seems to be quite essential for our purposes (in particular, for stating Lemma 3.3 below). It is worth recalling that this change of variables works, more in general, for equations like $u^{\prime \prime}+q(t) \mathfrak{g}(u)=0$, with $\mathfrak{g}(u)$ defined and never vanishing on an open interval $] \alpha, \beta[\subset \mathbb{R}$. From this point of view, while the results in [8] essentially deal with the highly asymmetric case $\mathfrak{g}^{\prime}(u) \rightarrow 0$ and $\mathfrak{g}^{\prime}(u) \rightarrow+\infty$ when $u \rightarrow \alpha^{+}$and $u \rightarrow \beta^{-}$, respectively (applying for instance to $\mathfrak{g}(u)=\exp (u)$, when ]$\alpha, \beta[=\mathbb{R}$ as in the present paper), our work can be viewed as a contribution to the case when $\mathfrak{g}^{\prime}(u) \rightarrow 0$ both for $u \rightarrow \alpha^{+}$and $u \rightarrow \beta^{-}$, which indeed yields a completely different dynamics. 
Let us finally mention that there is a rich bibliography dealing with BVPs for ordinary differential equations with indefinite weight. A significant part of this research, however, is concerned with the study of (oscillatory) solutions for (2) when $g(u)=\mathcal{G}^{\prime}(u)$, with $\mathcal{G}(u)>0$ for $|u|$ large (see, e.g., [10, 19, 21] and the references in $[7,8])$; these works are quite far in spirit from our investigation. As for $g(u)$ of constant sign on $\mathbb{R}$, we can quote the paper [17] as one of the few contributions available for the Neumann problem, dealing however with the case $g(u)=\exp (u)$ (see also [8]).

The paper is organized as follows. In Section 2 we recall the aforementioned change of variables and state our main results in the new coordinates. Moreover, we provide an illustrative strategy of the proofs, based on some numerical simulations involving the planar curves used in the shooting argument; the complete details are then given in Section 3. Finally, in Section 4 we discuss the optimality of our theorems, by showing a nonexistence result for $\mu \rightarrow 0^{+}$and $\mu \rightarrow+\infty$.

\section{Yet the main results: a more general point of view}

In this section, we present our main results under a slightly more general perspective. This is related to the change of variables (see [8]) mentioned at the end of the Introduction, which we now describe more in detail.

Let $g \in \mathcal{C}^{1}(\mathbb{R})$ satisfy

$$
g(u)>0 \quad \text { for every } u \in \mathbb{R}
$$

and

$$
\lim _{|u| \rightarrow+\infty} g^{\prime}(u)=0 .
$$

Of course, this is the case for both Theorems 1.1 and 1.2. Set

$$
W(u)=-\int_{0}^{u} \frac{d s}{g(s)}, \quad u \in \mathbb{R}
$$

in view of (11), $W(u)$ is a strictly decreasing $\mathcal{C}^{2}$-diffeomorphism of $\mathbb{R}$ onto its image. Moreover, (12) implies that $g(u) \leq|u|$ for $|u| \gg 0$, so that

$$
\int_{0}^{+\infty} \frac{d s}{g(s)}=+\infty=\int_{-\infty}^{0} \frac{d s}{g(s)},
$$

whence $W(\mathbb{R})=\mathbb{R}$. We can thus change variable in equation (2) by setting

$$
x(t)=W(u(t)),
$$

so as to obtain the second order equation (see also [20])

$$
x^{\prime \prime}=h(x)\left(x^{\prime}\right)^{2}+q(t),
$$


being $h: \mathbb{R} \rightarrow \mathbb{R}$ the continuous function given by

$$
h(x)=g^{\prime}\left(W^{-1}(x)\right), \quad x \in \mathbb{R} .
$$

Notice that, in view of (12), $h(x) \rightarrow 0$ for $|x| \rightarrow+\infty$; moreover, the uniqueness and the global continuability for the Cauchy problems associated with (14) are guaranteed since this is the case for (2). Since the Neumann conditions are clearly preserved by (13), we can solve the Neumann problem associated with (2) by finding solutions to (14) such that $x^{\prime}(0)=x^{\prime}(T)=0$. This is indeed the approach that we are going to follow henceforth.

To emphasize the role played by this change of variables, through the rest of the section we present our theorems directly referring to the second order differential equation (14), that is, we just assume that $h: \mathbb{R} \rightarrow \mathbb{R}$ is a continuous function such that

$$
\lim _{|x| \rightarrow+\infty} h(x)=0 .
$$

This gives a slightly wider point of view, since $h(x)$ does not need to be obtained from a function $g(u)$ as above ${ }^{2}$. It is worth noticing that the uniqueness for the Cauchy problems is still valid under the sole continuity of $h(x)$, as it is easily seen by writing the equivalent first order planar system

$$
X^{\prime}=Y \exp \left(\int_{0}^{X} h(s) d s\right), \quad Y^{\prime}=q(t) \exp \left(-\int_{0}^{X} h(s) d s\right),
$$

whose associated vector field is of class $\mathcal{C}^{1}$ in $(X, Y)$. On the other hand, the global continuability may fail and it will be explicitly assumed.

Taking $q(t)=q_{\mu}(t)$ as in (6) and recalling the definition of the critical value $\mu_{c}$ given in (8), we can now state our main results for the problem

$$
\left\{\begin{array}{l}
x^{\prime \prime}=h(x)\left(x^{\prime}\right)^{2}+\left(a^{+}(t)-\mu a^{-}(t)\right) \\
x^{\prime}(0)=x^{\prime}(T)=0
\end{array}\right.
$$

They can be seen, respectively, as more general versions of Theorems 1.1 and 1.2 in the Introduction, which indeed follow using the change of variables just described (with the choice $d=-W^{-1}(r)$, being $r$ as in the theorems below).

Theorem 2.1. Assume that $h \in \mathcal{C}(\mathbb{R})$ satisfies (15) and that the global continuability for the Cauchy problems associated with the differential equation in (16) is ensured (for any $\mu>0$ ). Moreover, suppose that, for a suitable $r>0$,

$$
h(x)>0 \quad \text { for }|x|>r .
$$

Finally, let $a \in L^{1}(0, T)$ satisfy (7). Then, there exists $\mu^{*}>\mu_{c}$ such that, for every $\mu \in] \mu_{c}, \mu^{*}[$, problem (16) has at least two solutions.

\footnotetext{
${ }^{2}$ This point requires some care. Indeed, as shown in [8], it is always possible to cast back equation (14) into an equation of the type $u^{\prime \prime}+q(t) \mathfrak{g}(u)=0$, but the function $\mathfrak{g}(u)$ may be possibly defined only on a proper open subinterval of $\mathbb{R}$.
} 
Theorem 2.2. Assume that $h \in \mathcal{C}(\mathbb{R})$ satisfies (15) and that the global continuability for the Cauchy problems associated with the differential equation in (16) is ensured (for any $\mu>0$ ). Moreover, suppose that, for a suitable $r>0$,

$$
\operatorname{sgn}(x) h(x)>0 \quad \text { for }|x|>r .
$$

Finally, let $a \in L^{1}(0, T)$ satisfy (7). Then, there exist $\mu_{*}, \mu^{*}>0$ with $\mu_{*}<\mu_{c}<\mu^{*}$ such that problem (16) has at least one solution for $\mu=\mu_{c}$ and two solutions for $\mu \in] \mu_{*}, \mu^{*}\left[\backslash\left\{\mu_{c}\right\}\right.$.

Similarly as in the second remark after Theorems 1.1 and 1.2, we have that the condition $\mu>\mu_{c}$ is necessary for the existence of a solution when $h(x)>0$ for any $x \in \mathbb{R}$ (see the proof of Lemma 3.1). Of course, this is possible only in the setting of Theorem 2.1.

We conclude this section by fixing some notation and giving an intuitive explanation of the shooting argument employed for the proofs, supported by some numerical simulations. The technical details are postponed to Section 3.

Let us consider, for any $\mu>0$, the first order planar system

$$
\left\{\begin{array}{l}
x^{\prime}=y \\
y^{\prime}=h(x) y^{2}+\left(a^{+}(t)-\mu a^{-}(t)\right)
\end{array}\right.
$$

and, for every $z_{0}=\left(x_{0}, y_{0}\right) \in \mathbb{R}^{2}$ and $t_{0} \in[0, T]$, denote by

$$
\zeta_{\mu}\left(\cdot ; t_{0}, z_{0}\right)=\left(x_{\mu}\left(\cdot ; t_{0}, z_{0}\right), y_{\mu}\left(\cdot ; t_{0}, z_{0}\right)\right)
$$

the solution to $(17)$ with $\zeta_{\mu}\left(t_{0} ; t_{0}, z_{0}\right)=z_{0}$. Moreover, define, for any $t_{0}, t_{1} \in[0, T]$, the application

$$
\left.\Phi^{t_{0}, t_{1}}:\right] 0,+\infty\left[\times \mathbb{R}^{2} \rightarrow \mathbb{R}^{2}, \quad\left(\mu, z_{0}\right) \mapsto \zeta_{\mu}\left(t_{1} ; t_{0}, z_{0}\right) .\right.
$$

The standard theory of ODEs guarantees that this map is continuous; moreover, for every $\mu>0$ fixed, $\Phi^{t_{0}, t_{1}}(\mu, \cdot)$ is a global diffeomorphism of the plane onto itself (the so-called Poincaré map). In the following, we will use the simplified notation $\Phi_{\mu}^{t_{0}, t_{1}}(\cdot)=\Phi^{t_{0}, t_{1}}(\mu, \cdot)$.

We now define the sets

$$
\Gamma^{+}=\Phi_{\mu}^{0, \tau}(\mathbb{R} \times\{0\})
$$

and

$$
\Gamma_{\mu}^{-}=\Phi_{\mu}^{T, \tau}(\mathbb{R} \times\{0\})
$$

and we notice that, in view of the previous discussion, they are unbounded $\mathcal{C}^{1}$-curves (parameterized by $x_{0} \in \mathbb{R}$ ) with no self-intersections. Moreover, the dependence of $\Gamma_{\mu}^{-}$on the parameter $\mu$ is continuous in the sense specified above, while, on the other hand, we have dropped the subscript $\mu$ from $\Gamma^{+}$since the map $\Phi_{\mu}^{0, \tau}$ does not depend on $\mu$ (indeed, $a^{-}(t)=0$ for $\left.t \in[0, \tau]\right)$. For this reason, in the following we simply write $\Phi^{0, \tau}$ (and, similarly, $\zeta\left(t ; t_{0}, z_{0}\right)$ instead of $\zeta_{\mu}\left(t ; t_{0}, z_{0}\right)$ if $\left.t, t_{0} \in[0, \tau]\right)$. 
It is now easily seen that $\zeta_{\mu}(t ; \tau, P)$ is a solution to (17) satisfying the Neumann boundary conditions $y_{\mu}(0 ; \tau, P)=y_{\mu}(T ; \tau, P)=0$ if and only if $P \in \Gamma^{+} \cap \Gamma_{\mu}^{-}$. Hence, we are led to look for intersection points between the curves $\Gamma^{+}$and $\Gamma_{\mu}^{-}$. Notice that, clearly, distinct intersections yield distinct Neumann solutions to (17).

The argument leading to Theorems 2.1 and 2.2 goes as follows. At first (see Lemma 3.3) we prove that

$(\star)$ the $y$-components of $\Gamma^{+}$and $\Gamma_{\mu}^{-}$, for $\left|x_{0}\right|$ large enough, are "near" $\int_{0}^{\tau} a^{+}(t) d t$ and $\mu \int_{\tau}^{T} a^{-}(t) d t$, respectively.

As a consequence, when $\mu=\mu_{c}$ the two curves $\Gamma^{+}$and $\Gamma_{\mu_{c}}^{-}$approach the same horizontal line when $\left|x_{0}\right| \rightarrow+\infty$. Focusing on the mutual position between $\Gamma^{+}$and $\Gamma_{\mu_{c}}^{-}$, we can also prove (see Lemma 3.5) the following:

(I) in the setting of Theorem 2.1, $\Gamma_{\mu_{c}}^{-}$lies definitively (for $\left|x_{0}\right|$ large) "below" $\Gamma^{+}$;

(II) in the setting of Theorem 2.2, $\Gamma_{\mu_{c}}^{-}$lies "above" $\Gamma^{+}$for $x_{0}$ negative and large and "below" $\Gamma^{+}$for $x_{0}$ positive and large.

These two situations are pictorially described in Figure 1.
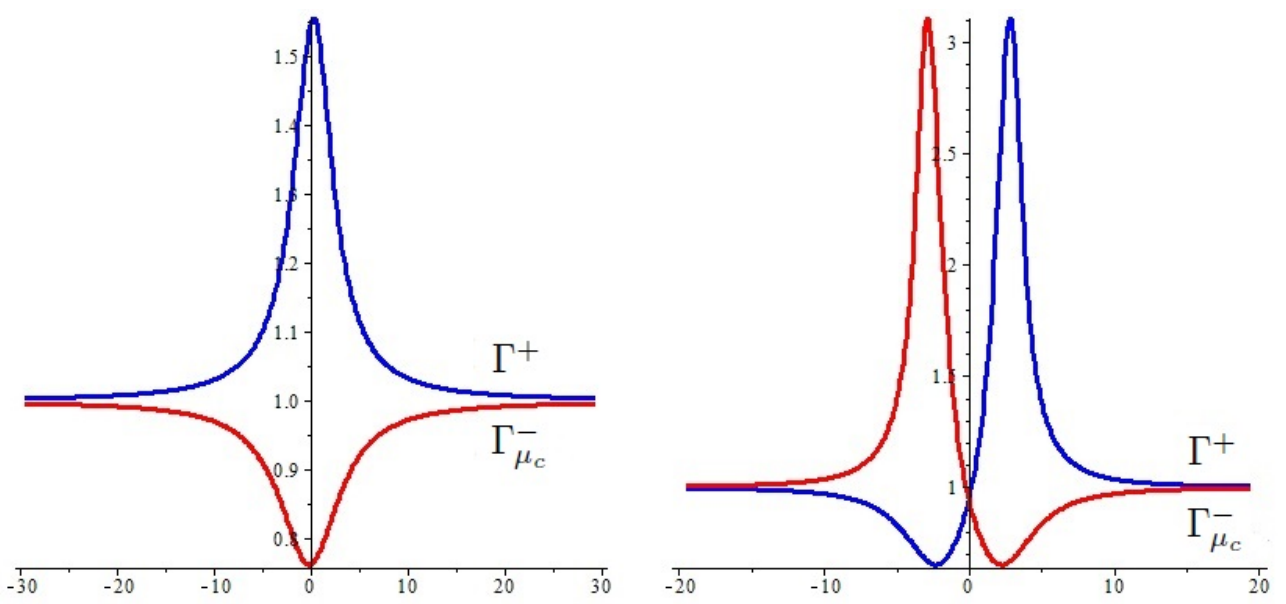

Figure 1: On the left, case (I) is depicted for $h(x)=1 /\left(1+0.1 x^{2}\right)$ and the piecewise constant weight function $a(t)=1$ on $[0, \tau[=[0,1[, a(t)=-1$ on $[\tau, T]=[1,2]$. On the right, we give a snapshot of case (II) for $h(x)=x /\left(1+0.01 x^{4}\right)$ and the same weight function $a(t)$. In both situations, $\mu=\mu_{c}=1$. Notice that, consistently with $(\star)$, the $y$-components of the curves $\Gamma^{+}$and $\Gamma_{\mu_{c}}^{-}$are near the value $1=\int_{0}^{\tau} a^{+}=\mu_{c} \int_{\tau}^{T} a^{-}$.

To conclude, we now have to analyze the behavior of the curves $\Gamma^{+}$and $\Gamma_{\mu}^{-}$ when $\mu$ varies in a neighborhood of $\mu_{c}$. In view of $(\star)$, the $y$-component of $\Gamma_{\mu}^{-}$ approaches $\mu \int_{\tau}^{T} a^{-}(t) d t$, so that we have the following situation:

(I) as soon as $\mu$ exceeds $\mu_{c}, \Gamma_{\mu}^{-}$climbs over $\Gamma^{+}$both for $x_{0}$ negative and large and for $x_{0}$ positive and large, giving (at least) two intersections for $\mu$ in a suitable right punctured neighborhood of $\mu_{c}$ (see Figure 2); 


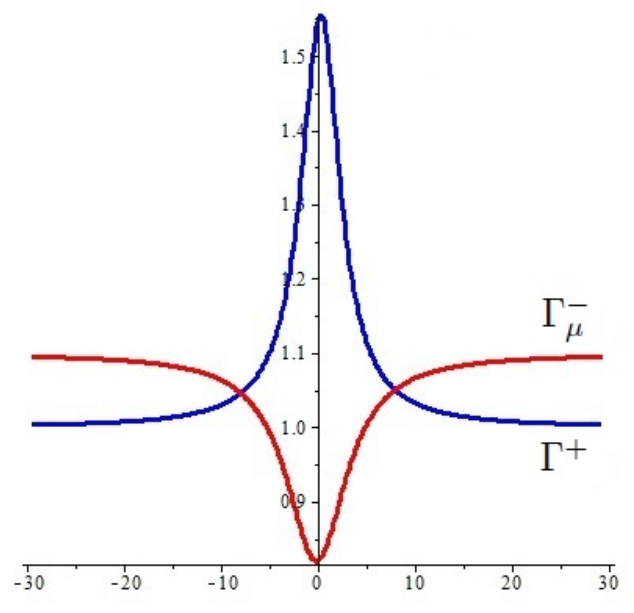

Figure 2: Here, case (I) is depicted (with $h(x)$ and $a(t)$ as in the left half of Figure 1) for $\mu=1.1>1=\mu_{c}$. Consistently with $(\star)$, the $y$-component of the curve $\Gamma_{\mu}^{-}$approaches the value $1.1=$ $\mu \int_{\tau}^{T} a^{-}$. Two intersection points (coming from $\pm \infty$ ) are found.

(II) for $\mu$ near $\mu_{c}, \Gamma_{\mu}^{-}$climbs under $\Gamma^{+}$for $x_{0}$ negative and large if $\mu<\mu_{c}$, and it climbs over $\Gamma^{+}$for $x_{0}$ positive and large if $\mu>\mu_{c}$. Thus, at least one intersection is found in a punctured neighborhood of $\mu_{c}$. On the other hand, the two curves $\Gamma^{+}$and $\Gamma_{\mu_{c}}^{-}$actually already intersect; clearly enough, this is true also for $\mu$ in a suitably small neighborhood of $\mu_{c}$, completing the picture.
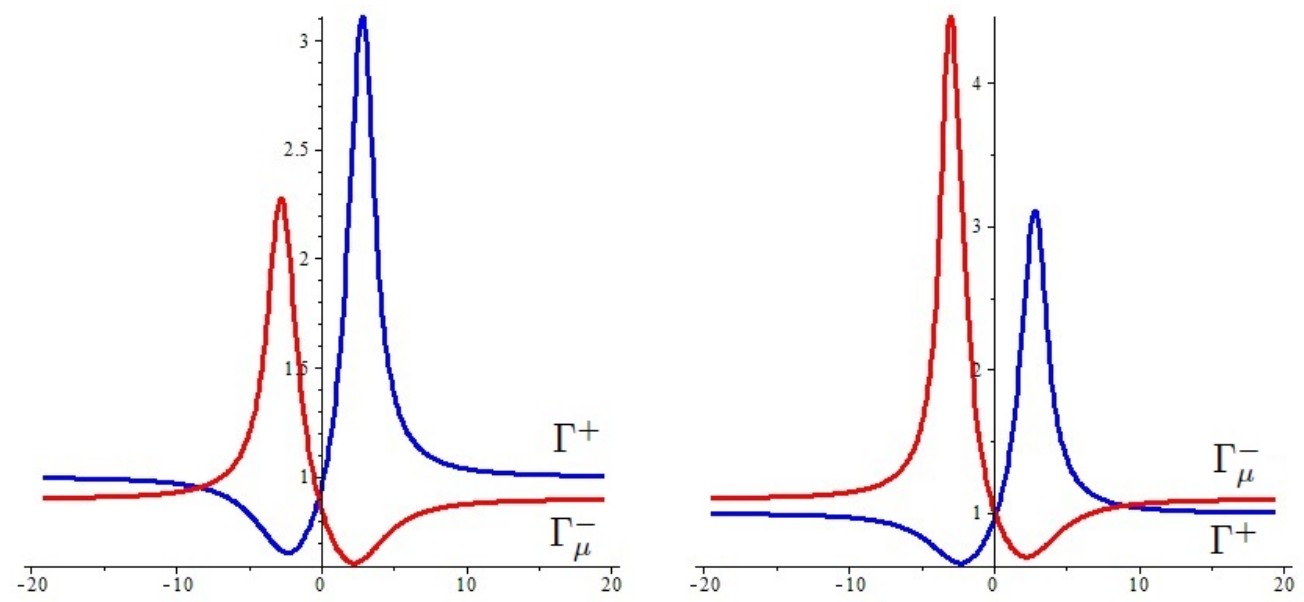

Figure 3: Here, case (II) is depicted (with $h(x)$ and $a(t)$ as in the right half of Figure 1) for $\mu=0.9<1=\mu_{c}$ on the left and $\mu=1.1>1=\mu_{c}$ on the right. Consistently with $(\star)$, the $y$-component of the curve $\Gamma_{\mu}^{-}$approaches the values 0.9 and 1.1, respectively. The "robust" intersection already present for $\mu=\mu_{c}$ (see Figure 1) is here preserved, while another intersection (coming from $-\infty$ on the left and from $+\infty$ on the right) is found.

A final remark: we are aware of the drawbacks of our numerical simulations, since we have not discussed the global continuability for the solutions giving rise to our plots (this is an assumption in our main results, and it appears as a non trivial matter when $h(x)$ has not been obtained from $g(u)$ via the above described 
change of variables). However, on one hand their purpose is purely illustrative; on the other hand, we think that they can be preferable with respect to handmade figures, since the solutions found numerically do not exhibit evidence of blow-up phenomena and seem to provide a nice insight into the dynamics of the considered equations.

\section{Proof of the main results}

In this section, we give the details of the informal argument described in Section 2 , thus providing the proofs of Theorems 2.1 and 2.2. We refer to the notations therein, as well as to the definition of the value $\mu_{c}$ given in (8).

To begin with, it is convenient to state a simple necessary condition for the existence of "large" solutions to (16). It relies on the basic assumption that the function $h(x)$ has constant sign definitively.

Proposition 3.1. Assume that there exists $r>0$ such that $h(x) \neq 0$ for every $|x|>r$ and let $x:[0, T] \rightarrow \mathbb{R}$ be a solution to (16) with $|x(t)|>r$ for every $t \in[0, T]$. Then $\mu \neq \mu_{c}$ and

$$
\operatorname{sgn}\left(\mu-\mu_{c}\right)=\operatorname{sgn}(h(x(t)))
$$

Proof. By integrating the differential equation in (16) and recalling (8), we get

$$
\begin{aligned}
\int_{0}^{T} h(x(t)) x^{\prime}(t)^{2} d t & =\mu \int_{\tau}^{T} a^{-}(t) d t-\int_{0}^{\tau} a^{+}(t) d t \\
& =\left(\mu-\mu_{c}\right) \int_{\tau}^{T} a^{-}(t) d t .
\end{aligned}
$$

Since $x^{\prime}(t) \not \equiv 0$ (otherwise $x(t) \equiv x(0)$, which is not a solution as $a(t) \not \equiv 0$ ) and $h(x(t)) \neq 0$ for every $t \in[0, T]$, the integral on the left hand-side is not zero, with $\operatorname{sign}$ equal to $\operatorname{sgn}(h(x(t)))$. The thesis follows.

It can be helpful to remark explicitly the consequences of Proposition 3.1 when $h(x)$ is a function satisfying the assumptions of Theorem 2.1 or Theorem 2.2. Namely, we have:

(I) if $h(x)>0$ for $|x|>r$, then no Neumann solutions $x:[0, T] \rightarrow \mathbb{R}$ with $x(t)>r$ or $x(t)<-r$ for every $t \in[0, T]$ can exist if $\mu \leq \mu_{c}$;

(II) if $\operatorname{sgn}(x) h(x)>0$ for $|x|>r$, then

- no Neumann solutions $x:[0, T] \rightarrow \mathbb{R}$ with $x(t)<-r$ for every $t \in[0, T]$ can exist if $\mu \geq \mu_{c}$,

- no Neumann solutions $x:[0, T] \rightarrow \mathbb{R}$ with $x(t)>r$ for every $t \in[0, T]$ can exist if $\mu \leq \mu_{c}$. 
Remark 3.2. Results of the type given in Proposition 3.1 can be formulated in the context (already discussed at the end of the Introduction) of an equation like

$$
u^{\prime \prime}+q(t) \mathfrak{g}(u)=0,
$$

with $\mathfrak{g}(u)$ a $\mathcal{C}^{1}$-function of constant sign defined on an open interval $] \alpha, \beta[\subset \mathbb{R}$ and such that $\mathfrak{g}^{\prime}(u)$ never vanishes on a subinterval $] \alpha^{\prime}, \beta^{\prime}[\subset] \alpha, \beta[$. Indeed, assuming that $u(t)$ is a Neumann solution to $(18)$ with $u(t) \in] \alpha^{\prime}, \beta^{\prime}[$ for every $t \in[0, T]$, dividing the equation by $\mathfrak{g}(u(t))$ and integrating (by parts) on $[0, T]$ we find

$$
\int_{0}^{T} q(t) d t=-\int_{0}^{T}\left(\frac{u^{\prime}(t)}{\mathfrak{g}(u(t))}\right)^{2} \mathfrak{g}^{\prime}(u(t)) d t .
$$

Hence

$$
\operatorname{sgn}\left(\int_{0}^{T} q(t) d t\right)=-\operatorname{sgn}\left(\left.\mathfrak{g}^{\prime}\right|_{\alpha^{\prime}, \beta^{\prime}[}\right),
$$

so that a mean value condition on the weight function appears as a necessary requirement for the solvability of the Neumann problem with solutions taking values in a preassigned interval (the reader is invited to compare with the statement and the proof of Proposition 3.1). Notice that the same happens if the T-periodic problem is considered, or if $u^{\prime \prime}$ is replaced by $\Delta u$ in a PDE setting. Conditions of this type were first introduced by Bandle, Pozio and Tesei [3], dealing with the existence of positive solutions to the Neumann problem for $\Delta u+q(x) u^{p}=0\left(x \in \Omega \subset \mathbb{R}^{N}\right)$, in the sublinear case $0<p<1$. In this setting, the necessary condition (19) leads to $\int_{\Omega} q(x) d x<0$ and it is shown to be sufficient for the (Neumann) solvability, as well. Afterwards, the role of mean value conditions for the solvability of BVPs associated with equations with a sign-changing weight function has been recognized and examined in several different frameworks (see, among others, $[1,5,8,9]$ and the bibliography in [7]).

Henceforth, we fix an open bounded neighborhood $U \subset] 0,+\infty\left[\right.$ of $\mu_{c}$ and we limit ourselves to consider values $\mu \in U$. This is not restrictive, since it is the case for our results. Our first lemma deals with the claim in $(\star)$, clarifying its meaning. Notice that it relies only on the fact that $h(x)$ is infinitesimal at infinity, while the sign condition does not play a role at this stage.

Lemma 3.3. Assume that $\lim _{|x| \rightarrow+\infty} h(x)=0$. Then, for every $\left.\left.\epsilon \in\right] 0,1\right]$, there exists $R_{\epsilon}>0$ such that

- for any $x_{0} \in \mathbb{R}$ with $\left|x_{0}\right| \geq R_{\epsilon}$,

$$
\left|y\left(\tau ; 0,\left(x_{0}, 0\right)\right)-\int_{0}^{\tau} a^{+}(t) d t\right|<\epsilon, \quad\left|x\left(\tau ; 0,\left(x_{0}, 0\right)\right)-x_{0}\right|<N,
$$

- for any $x_{0} \in \mathbb{R}$ with $\left|x_{0}\right| \geq R_{\epsilon}$ and for any $\mu \in U$,

$$
\left|y_{\mu}\left(\tau ; T,\left(x_{0}, 0\right)\right)-\mu \int_{\tau}^{T} a^{-}(t) d t\right|<\epsilon, \quad\left|x_{\mu}\left(\tau ; T,\left(x_{0}, 0\right)\right)-x_{0}\right|<N,
$$


with $N>0$ a suitable constant depending only on $a^{+}(t), a^{-}(t)$ and $U$.

Sketch of the proof. The proof is like the one of [8, Lemma 5]; for the reader's convenience, we provide a brief sketch. Set $c_{\xi}=\sup _{|x| \geq \xi}|h(x)|$ and, for $t \in[0, \tau]$,

$$
x(t)=x\left(t ; 0,\left(x_{0}, 0\right)\right), \quad z(t)=y\left(t ; 0,\left(x_{0}, 0\right)\right)-\int_{0}^{t} a^{+}(s) d s .
$$

For $\xi$ large, the differential inequality

$$
\left|z^{\prime}(t)\right| \leq c_{\xi}\left(z(t)+\int_{0}^{t} a^{+}(s) d s\right)^{2} \leq 2 c_{\xi}\left(z(t)^{2}+\left\|a^{+}\right\|_{L^{1}(0, \tau)}^{2}\right)
$$

implies that $|z(t)|$ is small as long as $t \in I=\{t \in[0, \tau]|| x(s) \mid \geq \xi$, for $s \in[0, t]\}$. As a consequence, $\left|x^{\prime}(t)\right|$ is bounded on $I$, so that $|x(t)| \geq \xi$ can actually be ensured when $\left|x_{0}\right|$ is large enough. Hence, $I=[0, \tau]$, so that $|z(\tau)|$ is small and $\left|x(\tau)-x_{0}\right|$ is bounded, which is the thesis.

The proof for the solutions on the time interval $[\tau, T]$ is analogous; of course, one has to use the fact that $\mu$ ranges in a bounded interval to make the constants $R_{\epsilon}$ and $N$ uniform with respect to the parameter.

Notice that, using the above arguments together with the continuity of the maps $\Phi^{t_{0}, t_{1}}$, we can assume (up to enlarging the constant $N$ ) that for any $x_{0} \in \mathbb{R}$ and $\mu \in U$,

$$
\left|x\left(t ; 0,\left(x_{0}, 0\right)\right)-x_{0}\right|<N \quad \text { for every } t \in[0, \tau] \text {, }
$$

and

$$
\left|x_{\mu}\left(t ; T,\left(x_{0}, 0\right)\right)-x_{0}\right|<N \quad \text { for every } t \in[\tau, T] .
$$

In particular, this implies that $\Gamma^{+}, \Gamma_{\mu}^{-}$are unbounded in the $x$-component. On the other hand, still from Lemma 3.3 we can deduce that they are bounded in their $y$-component.

Let us now consider the curve $\Gamma^{+}$. Since $\Phi^{0, \tau}$ is a global homeomorphism of $\mathbb{R}^{2}$ onto itself, $\Gamma^{+}$disconnects the plane into the two (pathwise) connected components

$$
\mathcal{O}^{u}=\Phi^{0, \tau}(\mathbb{R} \times] 0,+\infty[), \quad \mathcal{O}^{d}=\Phi^{0, \tau}(\mathbb{R} \times]-\infty, 0[) .
$$

Accordingly, the following holds true:

(o) if $\mathcal{S} \subset \mathbb{R}^{2}$ is a connected set, then one (and only one) of the following three possibilities occurs: $\mathcal{S} \subset \mathcal{O}^{d}, \mathcal{S} \subset \mathcal{O}^{u}$ or $\mathcal{S} \cap \Gamma^{+} \neq \emptyset$.

From this fact, it follows that any horizontal line which does not intersect $\Gamma^{+}$(recall that $\Gamma^{+}$is bounded in its $y$-component) is entirely contained either in $\mathcal{O}^{u}$ or in $\mathcal{O}^{d}$. By studying the direction of the vector field in (17) for $\left|y_{0}\right|$ large enough, we can easily deduce that $\mathcal{O}^{u}$ contains all the horizontal lines $y=y_{0}$ with $y_{0} \gg 0$, while $\mathcal{O}^{d}$ contains all the horizontal lines $y=y_{0}$ with $y_{0} \ll 0$. This gives a first justification to the superscripts $u$ and $d$, standing for "up" and "down", respectively. However, 
we need a more precise description of the sets contained in $\mathcal{O}^{u}, \mathcal{O}^{d}$, giving complete meaning to the terms "above" and "below" used in Section 2. To this aim, we introduce the following notation: for any $(\bar{x}, \bar{y}) \in \mathbb{R}^{2}$ we define the four cones

$$
\begin{aligned}
& \Sigma^{l, u}(\bar{x}, \bar{y})=\left\{(x, y) \in \mathbb{R}^{2} \mid x \leq \bar{x}, y \geq \bar{y}\right\}, \\
& \Sigma^{r, u}(\bar{x}, \bar{y})=\left\{(x, y) \in \mathbb{R}^{2} \mid x \geq \bar{x}, y \geq \bar{y}\right\}, \\
& \Sigma^{l, d}(\bar{x}, \bar{y})=\left\{(x, y) \in \mathbb{R}^{2} \mid x \leq \bar{x}, y \leq \bar{y}\right\}, \\
& \Sigma^{r, d}(\bar{x}, \bar{y})=\left\{(x, y) \in \mathbb{R}^{2} \mid x \geq \bar{x}, y \leq \bar{y}\right\} .
\end{aligned}
$$

Again, $u$ and $d$ stand for "up" and "down", while $l$ and $r$ stand for "left" and "right". In order to ease the reader's understanding, we give a pictorial representation of these cones in Figure 4 below.

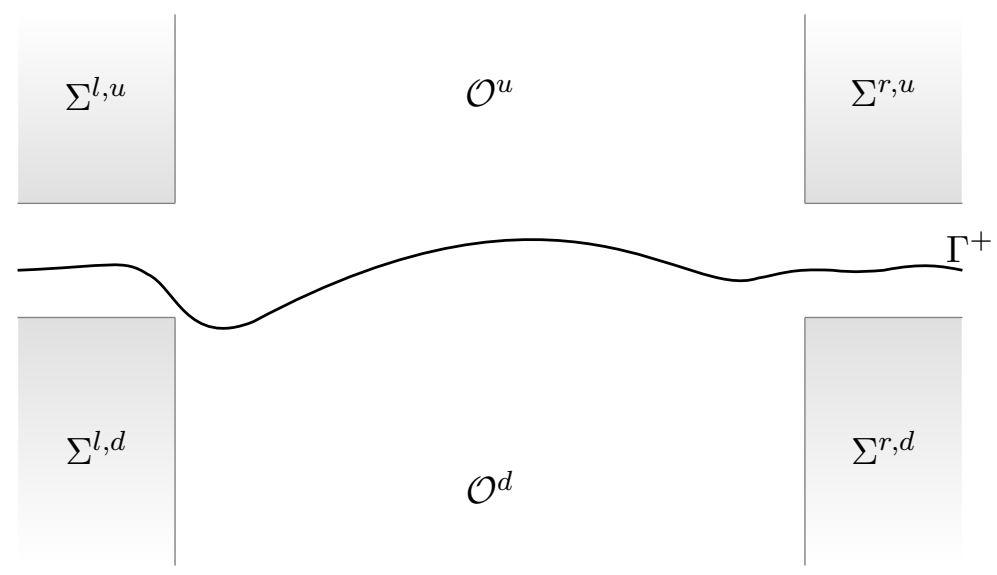

Figure 4: The four cones $\Sigma^{l, u}, \Sigma^{r, u}, \Sigma^{l, d}, \Sigma^{r, d}$ (not to overload the notation, we have omitted the point $(\bar{x}, \bar{y})$; of course, we have drawn $\Sigma^{l, u}, \Sigma^{r, u}$ for $\bar{y}>\int_{0}^{\tau} a^{+}(t) d t$, and $\Sigma^{l, d}, \Sigma^{r, d}$ for $\left.\bar{y}<\int_{0}^{\tau} a^{+}(t) d t\right)$.

We have the following result.

Lemma 3.4. For every $\bar{y} \neq \int_{0}^{\tau} a^{+}(t) d t$, there exists $\eta(\bar{y})>0$ such that

$$
\Sigma^{l, u}(-\eta(\bar{y}), \bar{y}) \cup \Sigma^{r, u}(\eta(\bar{y}), \bar{y}) \subset \mathcal{O}^{u} \quad \text { when } \quad \bar{y}>\int_{0}^{\tau} a^{+}(t) d t
$$

and

$$
\Sigma^{l, d}(-\eta(\bar{y}), \bar{y}) \cup \Sigma^{r, d}(\eta(\bar{y}), \bar{y}) \subset \mathcal{O}^{d} \quad \text { when } \quad \bar{y}<\int_{0}^{\tau} a^{+}(t) d t .
$$

Proof. We prove the thesis when $\bar{y}>\int_{0}^{\tau} a^{+}(t) d t$, the other case being analogous. Fix $\epsilon>0$ so small that

$$
\bar{y}>\int_{0}^{\tau} a^{+}(t) d t+\epsilon .
$$


Correspondingly, let $R_{\epsilon}$ be given by Lemma 3.3 and set $\eta(\bar{y})=R_{\epsilon}+N$ (which depends on $\bar{y}$ through $\epsilon)$. We claim that both $\Sigma^{l, u}(-\eta(\bar{y}), \bar{y})$ and $\Sigma^{r, u}(\eta(\bar{y}), \bar{y})$ are entirely contained in one connected component of $\mathbb{R}^{2} \backslash \Gamma^{+}$. Since they can be connected using an horizontal segment having large positive $y$-component, this ensures that $\Sigma^{l, u}(-\eta(\bar{y}), \bar{y})$ and $\Sigma^{r, u}(\eta(\bar{y}), \bar{y})$ are contained in the same connected component (recall (o)), and that this component is precisely $\mathcal{O}^{u}$.

We prove the claim for $\Sigma^{l, u}(-\eta(\bar{y}), \bar{y})$ (the argument for $\Sigma^{r, u}(\eta(\bar{y}), \bar{y})$ is completely symmetric and we will omit it for the sake of brevity). We write

$$
\Sigma^{l, u}(-\eta(\bar{y}), \bar{y})=\bigcup_{y \geq \bar{y}} \mathcal{L}^{l}(y)
$$

where, for a fixed $y \geq \bar{y}, \mathcal{L}^{l}(y)$ denotes the left half-line

$$
\mathcal{L}^{l}(y)=\left\{(x, y) \in \mathbb{R}^{2} \mid x \leq-\eta(\bar{y})\right\} .
$$

In view of (o), it is enough to prove that $\mathcal{L}^{l}(y) \cap \Gamma^{+}=\emptyset$ for any $y \geq \bar{y}$ (indeed, all the half-lines $\mathcal{L}^{l}(y)$ can be connected using the vertical half-line $\left.\{(-\eta(\bar{y}), y) \mid y \geq \bar{y}\}\right)$. To see this, define

$$
\left.\left.\gamma_{1}=\Phi^{0, \tau}(]-\infty,-R_{\epsilon}\right] \times\{0\}\right), \quad \gamma_{2}=\Phi^{0, \tau}\left(\left[-R_{\epsilon},+\infty[\times\{0\}),\right.\right.
$$

so that $\Gamma^{+}=\gamma_{1} \cup \gamma_{2}$, and fix $y \geq \bar{y}$. We are going to show that neither $\gamma_{1}$ nor $\gamma_{2}$ can intersect $\mathcal{L}^{l}(y)$ since their points have, respectively, different $y$-coordinate and different $x$-coordinate. More precisely:

- $\gamma_{1} \cap \mathcal{L}^{l}(y)=\emptyset$. Indeed, in view of the choice of $R_{\epsilon}$,

$$
\left.\gamma_{1} \subset \mathbb{R} \times\right] \int_{0}^{\tau} a^{+}(t) d t-\epsilon, \int_{0}^{\tau} a^{+}(t) d t+\epsilon[
$$

and the thesis follows from $(22)$, since $y \geq \bar{y}$.

- $\gamma_{2} \cap \mathcal{L}^{l}(y)=\emptyset$. Indeed, this is a consequence of $(20)$, since the $x$-component of any point in $\gamma_{2}$ is strictly greater than $-\left(R_{\epsilon}+N\right)=-\eta(\bar{y})$.

The proof is thus completed.

We now define, for any $\mu \in U$,

$$
\left.\left.\Gamma_{\mu}^{-, l}=\Phi_{\mu}^{T, \tau}(]-\infty,-(r+2 N)\right] \times\{0\}\right), \quad \Gamma_{\mu}^{-, r}=\Phi_{\mu}^{T, \tau}([r+2 N,+\infty[\times\{0\}),
$$

namely, the "left-tail" and the "right-tail" of $\Gamma_{\mu}^{-}$. Notice that, if $x=x_{\mu}:[0, T] \rightarrow \mathbb{R}$ is a solution to (16) with $\left|x_{\mu}(T)\right| \geq r+2 N$, then (20) and (21) imply that $\left|x_{\mu}(t)\right|>r$ for every $t \in[0, T]$. Then, Proposition 3.1 gives the following:

(I) if $h(x)>0$ for $|x|>r$, then

$$
\Gamma_{\mu}^{-, l} \cap \Gamma^{+}=\emptyset=\Gamma_{\mu}^{-, r} \cap \Gamma^{+} \quad \text { for every } \mu \leq \mu_{c} ;
$$


(II) if $\operatorname{sgn}(x) h(x)>0$ for $|x|>r$, then

$$
\Gamma_{\mu}^{-, l} \cap \Gamma^{+}=\emptyset \quad \text { whenever } \mu \geq \mu_{c}
$$

and

$$
\Gamma_{\mu}^{-, r} \cap \Gamma^{+}=\emptyset \quad \text { whenever } \mu \leq \mu_{c} .
$$

For $\mu=\mu_{c}$, we have in particular (both in case (I) and (II))

$$
\Gamma_{\mu_{c}}^{-, l} \cap \Gamma^{+}=\emptyset=\Gamma_{\mu_{c}}^{-, r} \cap \Gamma^{+} \text {. }
$$

Consequently, since $\Gamma_{\mu_{c}}^{-, l}, \Gamma_{\mu_{c}}^{-, r}$ are connected sets, (o) implies that each of them is contained in one of the connected components $\mathcal{O}^{u}, \mathcal{O}^{d}$. The next (crucial) lemma clarifies which situation can occur, according to the sign of $h(x)$ at infinity.

Lemma 3.5. The following hold true.

(I) Assume $h(x)>0$ for $|x|>r$. Then

$$
\Gamma_{\mu_{c}}^{-, l} \subset \mathcal{O}^{d} \quad \text { and } \quad \Gamma_{\mu_{c}}^{-, r} \subset \mathcal{O}^{d} .
$$

(II) Assume $\operatorname{sgn}(x) h(x)>0$ for $|x|>r$. Then

$$
\Gamma_{\mu_{c}}^{-, l} \subset \mathcal{O}^{u} \quad \text { and } \quad \Gamma_{\mu_{c}}^{-, r} \subset \mathcal{O}^{d}
$$

Proof. All the statements can be proved in the same way. For this reason, we give the details only for $\Gamma_{\mu_{c}}^{-, l}$ in case (I). By contradiction, assume that

$$
\Gamma_{\mu_{c}}^{-, l} \subset \mathcal{O}^{u}
$$

(notice that - as remarked before - we know that $\Gamma_{\mu_{c}}^{-, l}$ is entirely contained either in $\mathcal{O}^{d}$ or in $\mathcal{O}^{u}$, so that this is the logical negation of the thesis).

For $\mu \in U$, denote by $P_{\mu}$ the "endpoint" of $\Gamma_{\mu}^{-, l}$, i.e. $P_{\mu}=\Phi_{\mu}^{T, \tau}(-(r+2 N), 0)$. Since $P_{\mu_{c}} \in \Gamma_{\mu_{c}}^{-, l} \subset \mathcal{O}^{u}$ and $\mathcal{O}^{u}$ is open, the continuity of $\Phi_{\mu}^{T, \tau}$ yields the existence of $\mu<\mu_{c}$ such that $P_{\mu} \in \mathcal{O}^{u}$, implying

$$
\Gamma_{\mu}^{-, l} \cap \mathcal{O}^{u} \neq \emptyset .
$$

On the other hand, set

$$
\bar{y}=\frac{\mu+\mu_{c}}{2} \int_{\tau}^{T} a^{-}(t) d t\left(<\mu_{c} \int_{\tau}^{T} a^{-}(t) d t\right),
$$

and choose $\epsilon>0$ so small that

$$
\bar{y}+\epsilon<\mu_{c} \int_{\tau}^{T} a^{-}(t) d t\left(=\int_{0}^{\tau} a^{+}(t) d t\right) .
$$


For every $x_{0}<-\max \left\{R_{\epsilon}, \eta(\bar{y})+N\right\}$, we have

$$
\Phi_{\mu}^{T, \tau}\left(x_{0}, 0\right) \in \Sigma^{l, d}(-\eta(\bar{y}), \bar{y}) \subset \mathcal{O}^{d},
$$

where the first inclusion is guaranteed by Lemma 3.3 and the second one by Lemma 3.4. Taking, moreover, $x_{0} \leq-(r+2 N)$, we have $\Phi_{\mu}^{T, \tau}\left(x_{0}, 0\right) \in \Gamma_{\mu}^{-, l}$, so that

$$
\Gamma_{\mu}^{-, l} \cap \mathcal{O}^{d} \neq \emptyset .
$$

Recalling (o), (24) and (26) imply that $\Gamma_{\mu}^{-, l}$ has to intersect $\Gamma^{+}$, which is impossible in view of $(23)$ (being $\mu<\mu_{c}$ ).

We are now ready to complete the proofs of Theorems 2.1 and 2.2.

Proof of Theorem 2.1. In view of Lemma 3.5, case (I), and since $\mathcal{O}^{d}$ is open, the continuity of $\Phi_{\mu}^{T, \tau}$ allows us to choose $\mu^{*} \in U$, with $\mu^{*}>\mu_{c}$, such that, for any $\mu \in\left[\mu_{c}, \mu^{*}\left[\right.\right.$, the "left-endpoint" $P_{\mu}^{l}$ of $\Gamma_{\mu}^{-, l}$ (i.e., $P_{\mu}^{l}=\Phi_{\mu}^{T, \tau}(-(r+2 N), 0)$ ) as well as the "right-endpoint" $P_{\mu}^{r}$ of $\Gamma_{\mu}^{-, r}\left(P_{\mu}^{r}=\Phi_{\mu}^{T, \tau}(r+2 N, 0)\right)$ belong to $\mathcal{O}^{d}$. On the other hand, arguing as in the proof of Lemma 3.5, we can find points both of $\Gamma_{\mu}^{-, l}$ and of $\Gamma_{\mu}^{-, r}$ lying in $\mathcal{O}^{u}$, provided that $\left.\mu \in\right] \mu_{c}, \mu^{*}[$. Hence, (o) ensures that

$$
\left.\Gamma_{\mu}^{-, l} \cap \Gamma^{+} \neq \emptyset \neq \Gamma_{\mu}^{-, r} \cap \Gamma^{+} \quad \text { for every } \mu \in\right] \mu_{c}, \mu^{*}[.
$$

Since $\Gamma_{\mu}^{-, l}$ and $\Gamma_{\mu}^{-, r}$ are disjoint, we have found the two desired intersections.

Proof of Theorem 2.2. In view of Lemma 3.5, case (II), and since $\mathcal{O}^{u}$ is open, the continuity of $\Phi_{\mu}^{T, \tau}$ allows us to choose $\mu_{*} \in U$, with $\mu_{*}<\mu_{c}$, such that, for any $\mu \in] \mu_{*}, \mu_{c}\left[\right.$, the "left-endpoint" $P_{\mu}^{l}$ of $\Gamma_{\mu}^{-, l}$ (i.e., $P_{\mu}^{l}=\Phi_{\mu}^{T, \tau}(-(r+2 N), 0)$ ) of $\Gamma_{\mu}^{-, l}$ belongs to $\mathcal{O}^{u}$. On the other hand, arguing as in the proof of Lemma 3.5, we can find points of $\Gamma_{\mu}^{-, l}$ lying in $\mathcal{O}^{d}$, for any $\left.\mu \in\right] \mu_{*}, \mu_{c}[$. As a consequence, in view of (o),

$$
\left.\Gamma_{\mu}^{-, l} \cap \Gamma^{+} \neq \emptyset \quad \text { for every } \mu \in\right] \mu_{*}, \mu_{c}[\text {. }
$$

In a symmetric way, we can find $\mu^{*} \in U$, with $\mu^{*}>\mu_{c}$, such that the "rightendpoint" $P_{\mu}^{r}$ of $\Gamma_{\mu}^{-, r}$ belongs to $\mathcal{O}^{d}$ for $\mu \in\left[\mu_{c}, \mu_{*}[\right.$, and

$$
\left.\Gamma_{\mu}^{-, r} \cap \Gamma^{+} \neq \emptyset \quad \text { for every } \mu \in\right] \mu_{c}, \mu_{*}[.
$$

Summarizing, until now, we have found at least one intersection for $\mu \in] \mu_{*}, \mu^{*}\left[\backslash\left\{\mu_{c}\right\}\right.$.

We now define

$$
\Gamma_{\mu}^{-, K}=\Phi_{\mu}^{T, \tau}([-(r+2 N), r+2 N] \times\{0\}),
$$

that is, the compact connected subset of $\Gamma_{\mu}^{-}$obtained dropping its tails. From Lemma 3.5 and (o), it follows that

$$
\Gamma_{\mu_{c}}^{-, K} \cap \Gamma^{+} \neq \emptyset
$$


so that another intersection appears for $\mu=\mu_{c}$. Since, for any $\left.\mu \in\right] \mu_{*}, \mu^{*}[$, we have $P_{\mu}^{l} \in \mathcal{O}^{u}$ and $P_{\mu}^{r} \in \mathcal{O}^{d},(\circ)$ implies again that

$$
\left.\Gamma_{\mu}^{-, K} \cap \Gamma^{+} \neq \emptyset \quad \text { for every } \mu \in\right] \mu_{*}, \mu^{*}[\text {, }
$$

that is, we have a second intersection for $\mu$ in a neighborhood of $\mu_{c}$, clearly distinct from the one found before.

\section{Further remarks: nonexistence and dynamics in the phase-plane}

With the aim of discussing the optimality of our main results, in this final section we focus more in detail on the issue of nonexistence of solutions to

$$
\left\{\begin{array}{l}
u^{\prime \prime}+\left(a^{+}(t)-\mu a^{-}(t)\right) g(u)=0 \\
u^{\prime}(0)=u^{\prime}(T)=0
\end{array}\right.
$$

where $g \in \mathcal{C}^{1}(\mathbb{R})$ has constant (positive) sign and $a \in L^{1}(0, T)$ is as in (7).

We recall that some observations in this direction were already presented in the second remark after Theorems 1.1 and 1.2 and in Remark 3.2; precisely, problem (27) is never solvable for $\mu<\mu_{c}$ (resp., for $\mu>\mu_{c}$ ) whenever $g^{\prime}(u)>0$ (resp., $\left.g^{\prime}(u)<0\right)$ for every $u \in \mathbb{R}$. Notice that results of this type are useless when $g^{\prime}(u)$ changes sign (as in Theorem 1.2) and, in any case, they provide only one-sided intervals of non-solvability with respect to $\mu_{c}$. Hence, a more careful study of (27) for $\mu \rightarrow 0^{+}$and $\mu \rightarrow+\infty$ seems to be necessary.

A first, simple observation is the following: if the function $g(u)$ is bounded and bounded away from zero, namely, there exist $m, M>0$ such that $m \leq g(u) \leq M$ for any $u \in \mathbb{R}$, by integrating the equation on $[0, T]$ we easily see that a necessary condition for the solvability of (27) is that

$$
\frac{m}{M} \mu_{c} \leq \mu \leq \frac{M}{m} \mu_{c}
$$

This could be enough to show that our results, from the point of view of solvability in dependence on $\mu$, cannot be improved in general (and the value $\mu_{c}$ appears again to have a special role). However, if on one hand this simple remark applies to a wide class of nonlinearities $g(u)$ (independently of the assumptions on the derivative), on the other hand it requires some extra conditions on the range of $g(u)$ which are somewhat far from the approach followed in this paper. In particular, the hypothesis that $g(u)$ is bounded away from zero appears here unnatural, and indeed it is not satisfied by our model nonlinearities (4) and (5).

Our next result tries to fill this gap, showing that nonexistence for (27) when $\mu \rightarrow 0^{+}$and $\mu \rightarrow+\infty$ is a quite general fact. Precisely, it can always be proved whenever $g^{\prime}(u)$ is infinitesimal at infinity, provided that a slightly stronger assumption on $a(t)$ is fulfilled. 
Theorem 4.1. Let $g \in \mathcal{C}^{1}(\mathbb{R})$ be a positive function such that

$$
\lim _{|u| \rightarrow+\infty} g^{\prime}(u)=0 .
$$

Moreover, suppose that $a \in L^{1}(0, T)$ satisfies (7) and that $a^{-}(t)$ vanishes at most on a zero-measure subset of $[\tau, T]$. Then, there exist $\mu_{0}, \mu_{\infty}>0$, with $\mu_{0}<\mu_{c}<\mu_{\infty}$, such that problem (27) does not have a solution for $\mu \in] 0, \mu_{0}[\cup] \mu_{\infty},+\infty[$.

Proof. We first transform the differential equation in (27) into the system

$$
\left\{\begin{array}{l}
x^{\prime}=y \\
y^{\prime}=h(x) y^{2}+\left(a^{+}(t)-\mu a^{-}(t)\right)
\end{array}\right.
$$

via the change of variables described in Section 2, and notice that the uniqueness and the global continuability for (29) are guaranteed since they hold for (the differential equation in) (27). With the same notation as in Section 2, we thus consider the map

$$
\Phi_{\mu}^{t_{0}, t_{1}}\left(z_{0}\right)=\left(x_{\mu}\left(t_{1} ; t_{0}, z_{0}\right), y_{\mu}\left(t_{1} ; t_{0}, z_{0}\right)\right)
$$

(observe that here it is important to include the value $\mu=0$ in our considerations; of course, the continuity of $\Phi_{\mu}^{t_{0}, t_{1}}$ with respect to $\mu$ is guaranteed also in this case) and the curves $\Gamma^{+}$and $\Gamma_{\mu}^{-}$. Having in mind the shooting technique described in Section 2, this time we aim at showing that no intersections between $\Gamma^{+}$and $\Gamma_{\mu}^{-}$ can appear when $\mu$ is small or large. Precisely, we are going to prove that the projections of $\Gamma^{+}$and $\Gamma_{\mu}^{-}$on the $y$-axis have disjoint images.

As a preliminary step, we notice that, as shown in [8, Lemma 3], the curve $\Gamma^{+}$lies entirely in the open half-plane $\{y>0\}$. Since, in view of Lemma 3.3, $y\left(\tau ; 0,\left(x_{0}, 0\right)\right)$ converges to $\int_{0}^{\tau} a^{+}(t) d t>0$ for $\left|x_{0}\right| \rightarrow+\infty$, the continuity of $\Phi^{0, \tau}$ ensures then that there exist $c, C>0$ such that $c \leq y\left(\tau ; 0,\left(x_{0}, 0\right)\right) \leq C$ for every $x_{0} \in \mathbb{R}$ (here we have dropped the subscript since there is no dependence on $\mu$ ).

We first prove nonexistence for $\mu$ small, by showing that, when $\mu \rightarrow 0^{+}$, $y_{\mu}\left(\tau ; T,\left(x_{0}, 0\right)\right)<c$ for every $x_{0} \in \mathbb{R}$. To this aim, choose $\epsilon=c / 2$ and $\bar{\mu}=$ $c /\left(2 \int_{\tau}^{T} a^{-}(t) d t\right)$. Repeating the estimates in Lemma 3.3, we have that there exists $R_{\epsilon}>0$ such that, if $\left|x_{0}\right| \geq R_{\epsilon}$, then $\left|y_{\mu}\left(\tau ; T,\left(x_{0}, 0\right)\right)-\mu \int_{\tau}^{T} a^{-}(t) d t\right|<c / 2$ for $\mu \in[0, \bar{\mu}]$. Hence,

$$
\left|y_{\mu}\left(\tau ; T,\left(x_{0}, 0\right)\right)\right|<c \quad \text { for }\left|x_{0}\right| \geq R_{\epsilon}, \mu \in[0, \bar{\mu}] .
$$

On the other hand, by uniform continuity we have that

$$
\lim _{\mu \rightarrow 0^{+}} \Phi_{\mu}^{T, \tau}(\cdot, 0)=\Phi_{0}^{T, \tau}(\cdot, 0) \quad \text { uniformly for } x_{0} \in\left[-R_{\epsilon}, R_{\epsilon}\right]
$$

since $\Phi_{0}^{T, \tau}\left(x_{0}, 0\right)=\left(x_{0}, 0\right)$ for every $x_{0} \in \mathbb{R}$, this means that

$$
\lim _{\mu \rightarrow 0^{+}} y_{\mu}\left(\tau ; T,\left(x_{0}, 0\right)\right)=0 \quad \text { uniformly for } x_{0} \in\left[-R_{\epsilon}, R_{\epsilon}\right] .
$$


Consequently, (30) and (31) imply that, for $\mu$ sufficiently small, $y_{\mu}\left(\tau ; T,\left(x_{0}, 0\right)\right)<c$ for every $x_{0} \in \mathbb{R}$, as desired.

We now turn to the case when $\mu$ is large, by showing that, for $\mu \rightarrow+\infty$, $y_{\mu}\left(\tau ; T,\left(x_{0}, 0\right)\right)>C$ for every $x_{0} \in \mathbb{R}$. To this aim, assume, by contradiction, that there exist sequences $\mu_{k} \rightarrow+\infty$ and $x_{0}^{k} \in \mathbb{R}$ such that $y_{\mu_{k}}\left(\tau ; T,\left(x_{0}^{k}, 0\right)\right) \leq C$. Of course, two possibilities can occur: either $J_{k}=\sup _{t \in[\tau, T]}\left|y_{\mu_{k}}\left(t ; T,\left(x_{0}^{k}, 0\right)\right)\right|$ is bounded, or $\lim \sup _{k \rightarrow+\infty} J_{k}=+\infty$. In the following, we simply write $y_{\mu_{k}}(t)$ instead of $y_{\mu_{k}}\left(t ; T,\left(x_{0}^{k}, 0\right)\right.$ ) (an analogous notation is adopted for the $x$-component); moreover, we set $H=\max _{x \in \mathbb{R}}|h(x)|$.

- In the case $\widehat{J}=\sup _{k} J_{k}<+\infty$, using the second equation in (29) we have that

$$
\begin{aligned}
-C \leq-y_{\mu_{k}}(\tau) & =\int_{\tau}^{T}\left[h\left(x_{\mu_{k}}(t)\right) y_{\mu_{k}}(t)^{2}-\mu_{k} a^{-}(t)\right] d t \\
& \leq H \widehat{J}^{2}(T-\tau)-\mu_{k} \int_{\tau}^{T} a^{-}(t) d t
\end{aligned}
$$

which is a contradiction when $k$ is large.

- In the case when $\lim \sup _{k \rightarrow+\infty} J_{k}=+\infty$, we first observe that it has to be $\lim _{k \rightarrow+\infty} \sup _{t \in[\tau, T]} y_{\mu_{k}}(t)=+\infty$ (indeed, by a time-inversion argument, from $[8$, Lemma 4] it follows that $y_{\mu_{k}}(t) \geq 0$ for $\left.t \in[\tau, T]\right)$. It is then possible to choose an interval $\left[t_{k}^{-}, t_{k}^{+}\right] \subset[\tau, T[$ such that

$$
\left.y_{\mu_{k}}\left(t_{k}^{-}\right)=C, \quad y_{\mu_{k}}\left(t_{k}^{+}\right)=2 C, \quad C<y_{\mu_{k}}(t)<2 C \text { for every } t \in\right] t_{k}^{-}, t_{k}^{+}[.
$$

Using the second equation in (29), it follows that

$$
\begin{aligned}
C=y_{\mu_{k}}\left(t_{k}^{+}\right)-y_{\mu_{k}}\left(t_{k}^{-}\right) & =\int_{t_{k}^{-}}^{t_{k}^{+}}\left[h\left(x_{\mu_{k}}(t)\right) y_{\mu_{k}}(t)^{2}-\mu_{k} a^{-}(t)\right] d t \\
& \leq 4 H C^{2}\left(t_{k}^{+}-t_{k}^{-}\right)-\mu_{k} \int_{t_{k}^{-}}^{t_{k}^{+}} a^{-}(t) d t .
\end{aligned}
$$

As a consequence, $\left(t_{k}^{+}-t_{k}^{-}\right)$is bounded away from zero. Since, by assumption, $a^{-}(t)$ vanishes at most on zero measure subsets of $[\tau, T]$, we deduce that $\int_{t_{k}^{-}}^{t^{+}} a^{-}(t) d t$ is bounded away from zero, as well (observe that, up to subsequences, $t_{k}^{-}, t_{k}^{+}$converge). From

$$
C \leq 4 H C^{2}(T-\tau)-\mu_{k} \int_{t_{k}^{-}}^{t_{k}^{+}} a^{-}(t) d t,
$$

we thus get a contradiction when $k$ is large.

It is worth noticing that Theorem 4.1 applies to the model nonlinearities (4) (implying nonexistence for $\mu \rightarrow+\infty$; nonexistence for $\mu<\mu_{c}$ follows from the fact that $g^{\prime}(u)>0$ for every $u \in \mathbb{R}$ ) and (5) (implying nonexistence both for $\mu \rightarrow 0^{+}$ and for $\mu \rightarrow+\infty)$. Supported by this further piece of theoretical information, we 
conclude the paper by providing some numerical simulations for the dynamics of $u^{\prime \prime}+q_{\mu}(t) g(u)=0$ (with $g(u)$ as in (4) and (5)) in the standard phase-plane

$$
\left\{\begin{array}{l}
u^{\prime}=v \\
v^{\prime}=-q_{\mu}(t) g(u),
\end{array}\right.
$$

on varying of the parameter $\mu$. Similarly as in Section 2, we plot the two curves $\widetilde{\Gamma}^{+}$and $\widetilde{\Gamma}_{\mu}^{-}$obtained by shooting the $u$-axis, respectively, forward (from 0 to $\tau$ ) and backward (from $T$ to $\tau$ ) in time. The reader will certainly notice how subtler the situation appears to interpret, compared with the figures in Section 2.

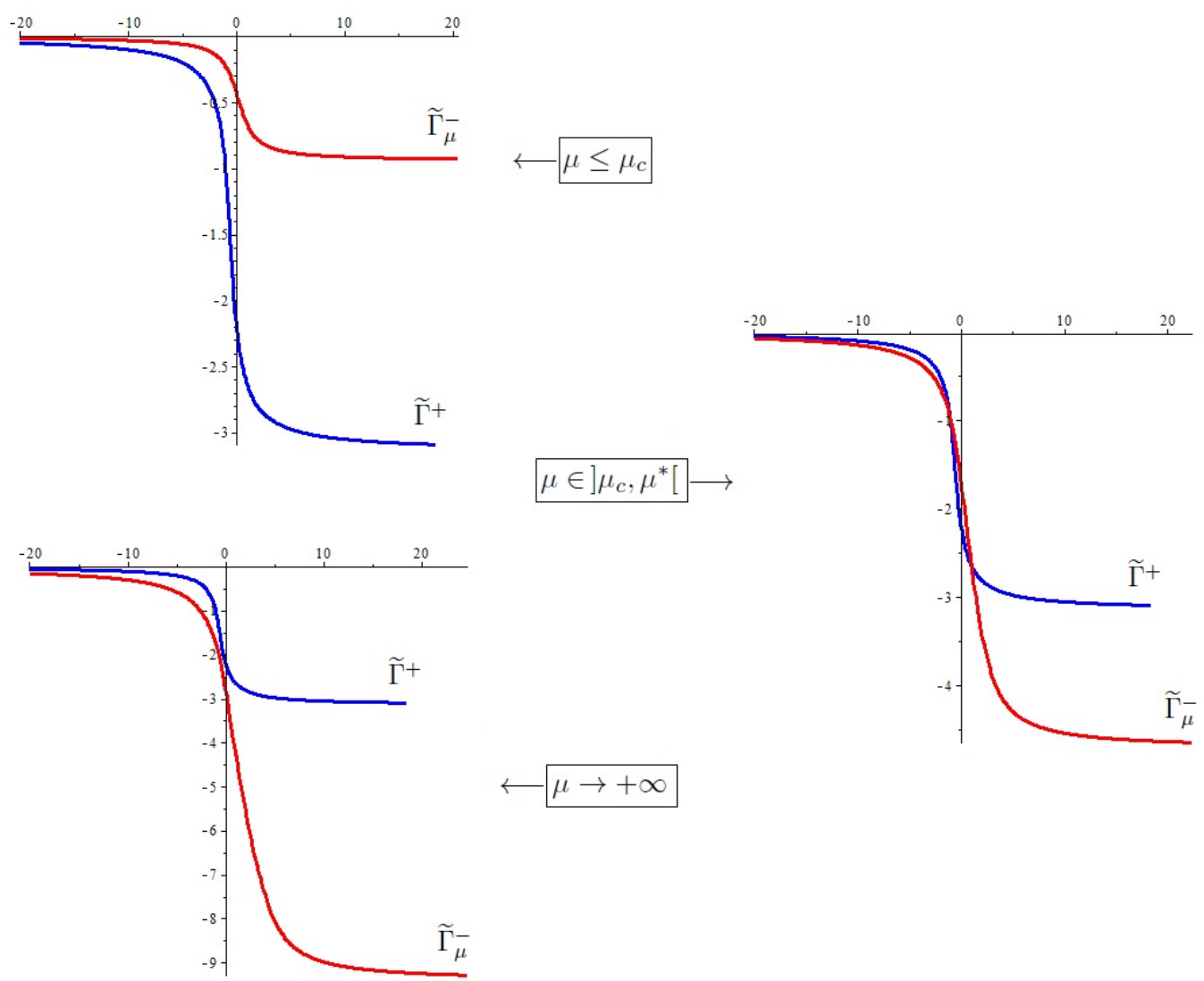

Figure 5: The dynamics in the standard phase-plane is depicted for case (I), with $g(u)=$ $\arctan (u)+\pi / 2$ and $a(t)=1$ on $[0, \tau[=[0,1[, a(t)=-1$ on $[\tau, T]=[1,2]$. In the figure, the parameter $\mu$ increases proceeding from the top to the bottom; the right half corresponds to values of $\mu$ (in the picture, $\mu=1.5$ ), greater than $\mu_{c}=1$, for which we have existence of at least two solutions, while in the left we have no solutions (for $\mu=0.3$, and $\mu=3$ ). In particular, consistently with Theorem 4.1 , we see that for $\mu \rightarrow 0^{+}$the curve $\widetilde{\Gamma}_{\mu}^{-}$lies entirely above $\widetilde{\Gamma}^{+}$, while for $\mu \rightarrow+\infty$ the opposite situation occurs. As for the existence, we recover the picture highlighted in Theorem 1.1. 


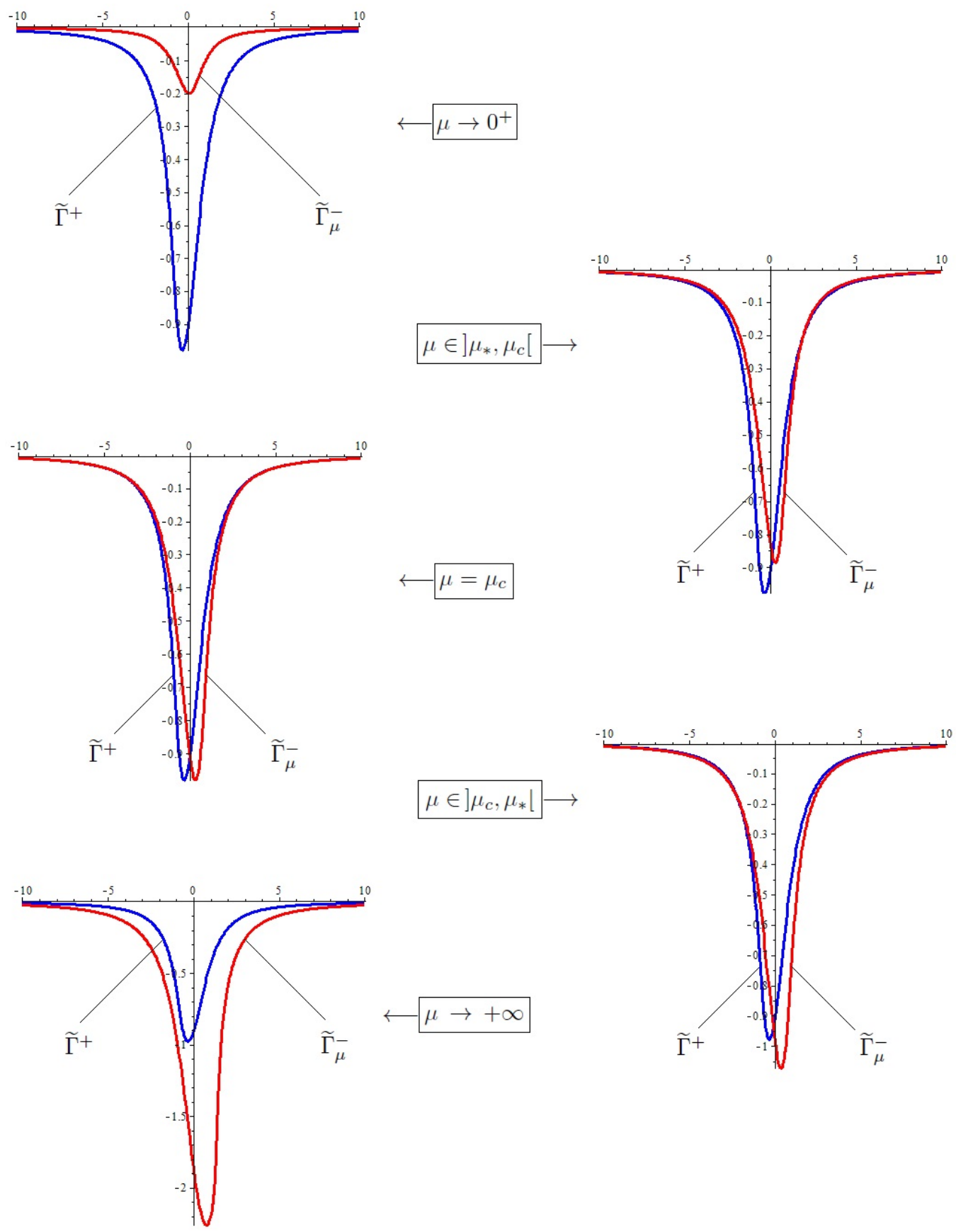

Figure 6: Here, the dynamics in the standard phase-plane is depicted for case (II), with $g(u)=1 /\left(1+u^{2}\right)$ and $a(t)=1$ on $[0, \tau[=[0,1[, a(t)=-1$ on $[\tau, T]=[1,2]$. In the figure, the parameter $\mu$ increases proceeding from the top to the bottom; the right half corresponds to values of $\mu$ (in the picture, $\mu=0.9$ and $\mu=1.1$ respectively) yielding at least two solutions, while in the left we have either no solutions (for $\mu=0.2$, and $\mu=2.5$ ) or, in general, at least one - but not necessarily more than one - in correspondence of the critical value $\mu_{c}$ (in our picture, $\mu_{c}=1$ ). Similarly as before, we see that for $\mu \rightarrow 0^{+}$the curve $\widetilde{\Gamma}_{\mu}^{-}$lies entirely above $\widetilde{\Gamma}^{+}$, while for $\mu \rightarrow+\infty$ the opposite situation occurs. As for the existence, we recover the picture highlighted in Theorem 1.2 (notice that for $\mu=\mu_{c}$ we only see an intersection between $\widetilde{\Gamma}^{+}$and $\widetilde{\Gamma}_{\mu}^{-}$). 


\section{References}

[1] S. Alama and G. Tarantello, On semilinear elliptic equations with indefinite nonlinearities, Calc. Var. Partial Differential Equations 1 (1993), 439-475.

[2] F.V. Atkinson, W.N. Everitt and K.S. Ong, On the m-coefficient of Weyl for a differential equation with an indefinite weight function, Proc. London Math. Soc. (3) 29 (1974), 368-384.

[3] C. Bandle, M.A. Pozio and A. Tesei, Existence and uniqueness of solutions of nonlinear Neumann problems, Math. Z. 199 (1988), 257-278.

[4] C. Bereanu and J. Mawhin, Multiple periodic solutions of ordinary differential equations with bounded nonlinearities and $\varphi$-Laplacian, NoDEA Nonlinear Differential Equations Appl. 15 (2008), 159-168.

[5] H. Berestycki, I. Capuzzo-Dolcetta and L. Nirenberg, Variational methods for indefinite superlinear homogeneous elliptic problems, NoDEA Nonlinear Differential Equations Appl. 2 (1995), 553-572.

[6] D. Bonheure, J.M. Gomes and P. Habets, Multiple positive solutions of superlinear elliptic problems with sign-changing weight, J. Differential Equations 214 (2005), 36-64.

[7] A. Boscaggin and F. Zanolin, Pairs of positive periodic solutions of second order nonlinear equations with indefinite weight, J. Differential Equations 252 (2012), 2900-2921.

[8] A. Boscaggin and F. Zanolin, Second order ordinary differential equations with indefinite weight: the Neumann boundary value problem, Ann. Mat. Pura Appl. (4), online first.

[9] J.L. Bravo and P.J. Torres, Periodic solutions of a singular equation with indefinite weight, Adv. Nonlinear Stud. 10 (2010), 927-938.

[10] G.J. Butler, Rapid oscillation, nonextendability, and the existence of periodic solutions to second order nonlinear ordinary differential equations, J. Differential Equations 22 (1976), 467-477.

[11] J.Á. Cid and L. Sanchez, Periodic solutions for second order differential equations with discontinuous restoring forces, J. Math. Anal. Appl. 288 (2003), 349-364.

[12] G. Feltrin and F. Zanolin, Multiple positive solutions for a superlinear problem: a topological approach, preprint.

[13] A. Fonda and M. Garrione, Nonlinear resonance: a comparison between Landesman-Lazer and Ahmad-Lazer-Paul conditions, Adv. Nonlinear Stud. 11 (2011), 391-404. 
[14] M. Gaudenzi, P. Habets and F. Zanolin, An example of a superlinear problem with multiple positive solutions, Atti Sem. Mat. Fis. Univ. Modena 51 (2003), 259-272.

[15] P.M. Girão and J.M. Gomes, Multibump nodal solutions for an indefinite superlinear elliptic problem, J. Differential Equations 247 (2009), 1001-1012.

[16] P, Hess and T. Kato, On some linear and nonlinear eigenvalue problems with an indefinite weight function, Comm. Partial Differential Equations 5 (1980), 999-1030.

[17] V.K. Le and K. Schmitt, Minimization problems for noncoercive functionals subject to constraints, Trans. Amer. Math. Soc. 347 (1995), 4485-4513.

[18] J. Mawhin and M. Willem, Critical Point Theory and Hamiltonian Systems, Applied Mathematical Sciences 74, Springer-Verlag, New York, 1989.

[19] D. Papini and F. Zanolin, A topological approach to superlinear indefinite boundary value problems, Topol. Methods Nonlinear Anal. 15 (2000), 203-233.

[20] M. Sabatini, On the period function of $x^{\prime \prime}+f(x) x^{\prime 2}+g(x)=0$, J. Differential Equation 196 (2004), 151-168.

[21] S. Terracini and G. Verzini, Oscillating solutions to second-order ODEs with indefinite superlinear nonlinearities, Nonlinearity 13 (2000), 1501-1514.

[22] J.R. Ward, Periodic solutions of ordinary differential equations with bounded nonlinearities, Topol. Methods Nonlinear Anal. 19 (2002), 275-282.

Authors' addresses:

Alberto Boscaggin

Dipartimento di Matematica, Università di Torino, Via Carlo Alberto 10, I-10123 Torino, Italy

e-mail: alberto.boscaggin@unito.it

Maurizio Garrione

Dipartimento di Matematica e Applicazioni, Università di Milano-Bicocca, Via Cozzi 53, I-20125 Milano, Italy

e-mail: maurizio.garrione@unimib.it 